\title{
A bilateral optic neuritis side effect of biological treatment with pembrolizumab in a patient with metastatic melanoma
}

\begin{abstract}
Pembrolizumab is an anti-PD-1 humanized monoclonal antibody, standardized therapy for the treatment of metastatic melanoma, providing an overall survival benefit to patients; however, it may be associated with immune-related adverse effects (irAE). We describe the case of a patient with a history of metastatic melanoma undergoing treatment with pembrolizumab, who presented visual acuity deterioration due to inflammatory involvement of the optic nerves. There are few reported cases of an optic neuritis side effect of treatment with immune checkpoint inhibitors. The patient received high doses of steroids and plasmapheresis with improved light perception. This case highlights the need to recognize atypical processes, mediated by the immune system, associated with treatment with immune checkpoint inhibitors (ICI), including pembrolizumab
\end{abstract}

Keywords: melanoma, pembrolizumab, optic neuritis, immune checkpoint inhibitors, immune-related adverse effects
Volume 10 Issue 5 - 2020

\author{
Andrea Nassar,' Neiby Rivera,' Javier Mora,' \\ Ayleen Martinez ${ }^{3}$ \\ 'Neurologist specialist, Department of Internal Medicine, \\ Hospital Universitario clínica San Rafael, Colombia \\ ${ }^{2}$ Internal Medicine specialist, Department of Internal Medicine, \\ Fundación Universitaria Juan N. Corpas, Hospital Universitario \\ clínica San Rafael, Colombia \\ ${ }^{3}$ Internal Medicine Resident, Department of internal Medicine, \\ Fundación Universitaria Juan N. Corpas, Hospital Universitario \\ Clinica San Rafael, Colombia
}

Correspondence: Andrea Nassar, Department of Neurology of Hospital Universitario Clínica San Rafael, Carrera 8 \# 17-45 Sur, Bogotá D.C, Colombia,

Email andrea-nassar@juanncorpas.edu.co

Received: September 27, 2020 | Published: October 14, 2020

\begin{abstract}
Abbreviations: ON, optic neuritis; MS, multiple sclerosis; NMO, neuromyelitis optica; CRION, idiopathic recurrent chronic optic neuritis; PDE5, phosphodiesterase type 5 inhibitors specific to cyclic guanosine monophosphate; CTLA-4, cytotoxic T-lymphocyte; irAE, antigen 4 immune-related adverse effects; IVIG, intravenous immunoglobulin; CSF, cerebrospinal fluid; ANA, Antinuclear antibody; ICI, Immune checkpoint inhibitors; CNS, central nervous system; ICP, intracranial pressure; MRI, brain magnetic resonance imaging
\end{abstract}

\section{Introduction}

Advanced melanoma is an aggressive skin cancer tumor, which has been characterized by low survival and poor response to cytotoxic chemotherapy; ${ }^{1}$ it is the fifth most common skin cancer among men and the sixth among women ${ }^{2}$ however, mortality has decreased due to early detection and availability of treatments for advanced tumors. ${ }^{3}$ These new drugs have evidenced significant benefits in overall survival. ${ }^{4-6}$

Since 2011, the Food and Drug Administration of the United States has been approving new treatments for advanced unrespectable melanoma: cytotoxic T-lymphocyte antigen 4 (CTLA-4) antibody, ipilimumab, the targeted BRAF inhibitor, vemurafenib, ${ }^{7-11}$ and antiPD-1 humanized monoclonal antibodies, such as pembrolizumab and nivolumab. ${ }^{12-15}$ These state-of-the-art treatments have evidenced improvement and long-lasting response.

However, they can be associated with immune-related adverse effects (irAE) by causing an immune system imbalance between host T cells and tumor cells, which can increase the anti-tumor T cell response in some cases. ${ }^{16}$ These respond well to steroid treatment; however, there are refractory cases that require intravenous immunoglobulin (IVIG). Ophthalmological complications of treatment with immune checkpoint inhibitors are rare, ${ }^{16}$ they have been described in less than $1 \%$ of patients. ${ }^{17-19}$ Blurred vision, decreased visual acuity, dry eye, eye pain, and photophobia may occur. ${ }^{19,20}$ We present the case of a patient with metastatic melanoma with atypical optic neuritis associated with the use of pembrolizumab.

\section{Clinical case}

Female patient in her seventh decade of life with a history of postsurgical hypothyroidism due to surgically treated papillary thyroid cancer, bilateral cataract, and metastatic melanoma undergoing treatment with pembrolizumab every 21 days, who was admitted to the emergency department for rapid and insidious visual acuity deterioration up to blindness, associated with eye pain; to neurological assessment with bilateral lens opacity with bilateral amaurosis and the rest of the neurological examination without abnormalities, fluorescein retinal angiography of both eyes, which evidenced optic neuritis of the right eye (Figure 1), which a probable autoimmune phenomenon associated with paraneoplastic syndrome was suspected, versus adverse effect related to the immune system (irAE) due to pembrolizumab.

During hospitalization, the following were performed: hemogram, creatinine, BUN, electrolytes without abnormalities, extended examination with infectious panel (serology, hepatitis B and C, and HIV), immune panel: Antinuclear antibody (ANA) 1:160 speckled pattern (serum), anti-dsDNA, Anti-SCL-70, P-Anca, and C- Anca, myeloperoxidase, and including ANA and flow cytometry in CSF were normal. Performed a simple orbital resonance with a contrast medium (Figure 2) that showed an abnormality in the signal and 
enhancement of the optic chiasm, optic radiations and optic nerves bilaterally, conclusive findings of bilateral longitudinally extensive optic neuritis.

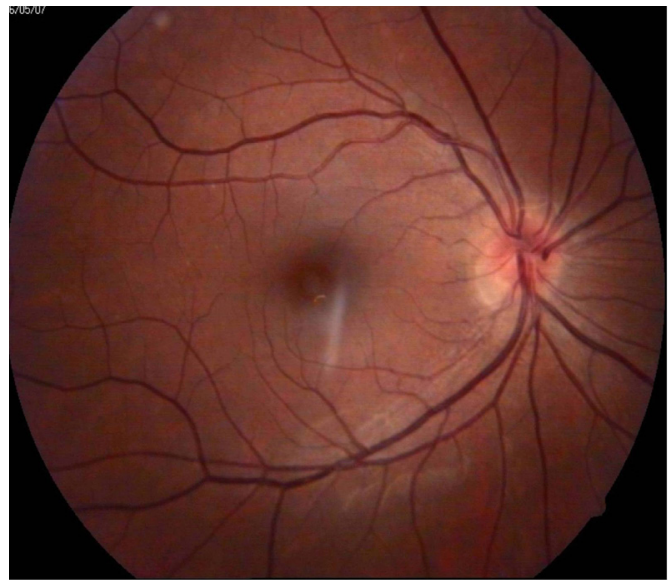

Figure I Fluorescein retinal angiography of both eyes showing optic neuritis of the right eye.
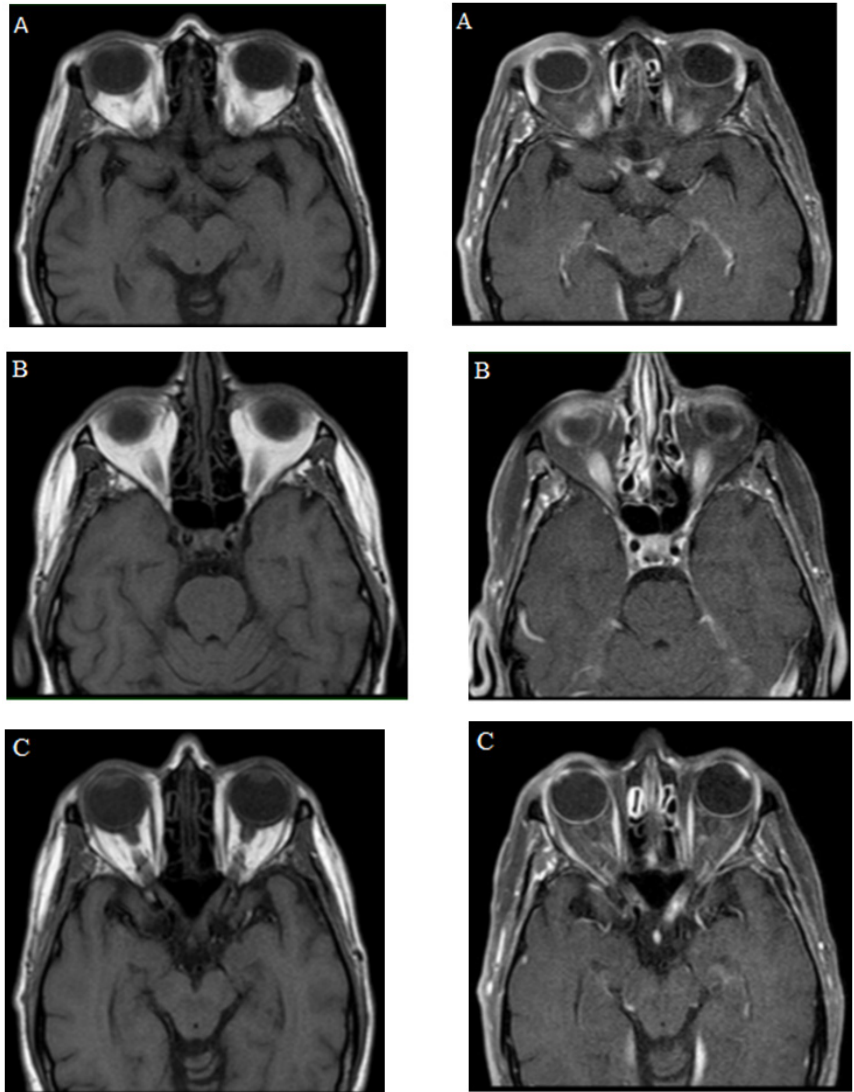

Figure 2 Simple MRI of orbits and with a medium of contrast. Simple and contrasted $\mathrm{TI}$ images, $(\mathrm{A}, \mathrm{B}$, and $\mathrm{C})$ evidencing signal alteration and enhancement with the medium of contrast, of the optic chiasm, optic radiations, and optic nerves, characteristic findings of longitudinally extensive bilateral optic neuritis.

Because of optic nerve involvement, without any other documentable etiology in the paraclinical admission, the most likely cause was considered to be a side effect of pembrolizumab, which is why it was discontinued. The patient was treated with methylprednisolone 1 gram per day for 5 days without improvement, requiring treatment with plasmapheresis completing 7 days, with subtle improvement of light perception in the right eye. The patient was discharged and assessed 30 days after checkup without exhibiting clinical deterioration. Partial conduction in the optic nerves was evidenced in the visual evoked potentials taken at discharge (Figure 3). She was referred to undergo a bilateral phakectomy. She is currently scheduled for surgery.

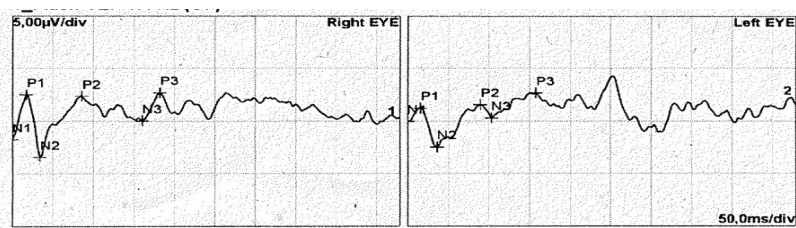

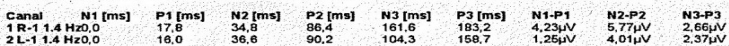

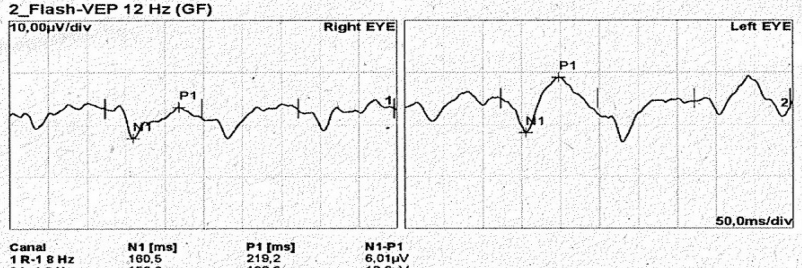

Figure 3 Visual evoked potentials, showing partial conduction of the optic nerves.

\section{Discussion}

Advanced melanoma is an aggressive skin cancer tumor, characterized by a low survival rate and poor response to cytotoxic chemotherapy. ${ }^{1}$ It is the fifth most common skin cancer among men and the sixth among women. ${ }^{2}$ Incidence continues to rise, with a risk of 1 in 24 people; however, mortality has decreased due to early detection and availability for advanced tumors treatment. ${ }^{3}$ Novel agents such as anti-PD-1 humanized monoclonal antibodies like nivolumab and pembrolizumab have shown significant antitumor activity in a variety of cancers improving survival as in the case of patients with advanced melanoma. ${ }^{1,3}$

Immune checkpoint inhibitors (ICI) are drugs indicated for some types of cancers such as melanoma, lung cancer, mesothelioma, kidney cancer, lymphoma, and metastatic prostate cancer, because of their anti-cytotoxic effect associated with T-4 lymphocytes (CTLA4), which induce various cytokines, as well as activate PD-L1 to PD-1 in T cells, and inhibit T cell proliferation and production of proinflammatory Th1 cytokines, including IFN- $\gamma$ and IL-2. This cascade reaction activates the PD-1/PD-L1 pathway, which plays a critical role in controlling T-cell activity in inflamed tissues.

Antibodies to PD-1, such as nivolumab, pembrolizumab, and pidilizumab prevent T-cell inactivation by blocking the PD-1/PDL1 pathway between host T-cells and tumor cells, which eventually increases antitumor T-cell response; however, by causing an imbalance in the immune system, these new immunotherapies also generate dysimmune toxicities, denominated immune-related adverse effects (irAE) that primarily involve the intestine, skin, liver, endocrine glands, lungs, and nervous system and can, therefore, affect any organ. ${ }^{21}$ Among patients experiencing irAEs, 1-5\% show neurological dysfunction and less than $1 \%$ have ophthalmological complications. ${ }^{21,22}$ Recently, there was a report of optic neuritis together with treatment with anti-CTLA $4^{23}$ antibodies; however, a 
detailed description of optic neuritis associated with treatment with anti-PD-1 or anti-PD-L1 antibodies has not yet been published. ${ }^{24}$

Optic neuritis $(\mathrm{ON})$ is a mediated inflammatory optic neuropathy. Most typical cases are unilateral and mainly affect young caucasian women. The main symptoms are vision loss, pain with eye movement and dyschromatopsia. The ocular fundus is regularly normal and neuroimaging may vary in its characteristics according to etiology. ${ }^{25}$ ON can be classified according to its etiology (Table 1) or its form of presentation, in typical or atypical (Table 2).

Table I Causes of typical and atypical optic neuritis

\begin{tabular}{|c|c|c|}
\hline Etiology & Typical & Atypical \\
\hline \multirow{2}{*}{ Ischemic } & Arteritic & \multirow{2}{*}{ Not Applicable } \\
\hline & Non-Arteritic & \\
\hline \multirow{3}{*}{ Toxic/Metabolic } & & lonizing Radiation \\
\hline & & Pharmacons \\
\hline & & Deficiency:Vitamin BI2 Deficit \\
\hline \multirow{5}{*}{ Infectious } & & Syphilis \\
\hline & & Tuberculosis \\
\hline & & Lyme disease, \\
\hline & & HIV \\
\hline & & CMV \\
\hline \multirow{3}{*}{ Parainfectious/Autoimmune } & Neuromyelitis Optica & Postinfection \\
\hline & \multirow[t]{2}{*}{ Multiple Sclerosis } & Postvaccinal \\
\hline & & For neoplastic: Solid Intracranial Tumors, \\
\hline \multirow{5}{*}{ Inflammatory/Vasculitis } & & Sarcoidosis \\
\hline & & Granulomatous Vasculitis \\
\hline & & SLE \\
\hline & & Sjögren \\
\hline & & Behçet \\
\hline \multirow{4}{*}{ Neoplastic } & & Optic Nerve Tumors \\
\hline & & Intracranial Mass Metastases \\
\hline & & Abscesses \\
\hline & & Aneurysms \\
\hline Hereditary & & Leber's Optic Neuropathy \\
\hline Others & Idiopathic & \\
\hline
\end{tabular}

Table 2 Findings of typical and atypical optic neuritis

\begin{tabular}{|c|c|}
\hline Typical & Atypical \\
\hline \multicolumn{2}{|l|}{ Clinical manifestations } \\
\hline Unilateral & Bilateral \\
\hline Woman & Man \\
\hline $\begin{array}{l}\text { Significant eye pain that may wake the patient up while sleeping } \\
\text { or last more than } 2 \text { weeks }\end{array}$ & May or may not have eye pain \\
\hline Average age 32 years old & Under 12 years old, over 50 years old \\
\hline Normal optic nerve in $2 / 3$ of patients, optic nerve edema in $1 / 3$ & Significant edema of the optic nerve, chiasm, and optic tracts. \\
\hline Clinical improvement usually within the first 4 weeks & Progressive vision loss after more than two weeks \\
\hline More dyschromatopsia than loss of visual acuity & Visual activity $20 / 200$ or worse \\
\hline \multicolumn{2}{|l|}{ Medical imaging findings } \\
\hline $\begin{array}{l}\text { Anterior enhancement of the nerve that does not compromise } \\
\text { more than } 1 / 3 \text { of the optic nerve }\end{array}$ & $\begin{array}{l}\text { Enhancement of the optic nerve that may be greater than } 2 / 3 \text { of the } \\
\text { nerve, compromised optic chiasm and tracts. }\end{array}$ \\
\hline
\end{tabular}


Typical ON is associated in a significant percentage to multiple sclerosis (MS) ${ }^{26}$ for this case, it is generally unilateral, presents itself in young, Caucasian women. Clinically, it manifests with eye pain, dyschromatopsia, decreased visual acuity, scotoma, normal papilla, or with slight edema, it responds to treatment with high doses of intravenous steroid and during neuroimaging, demyelinating lesions are found, typical of MS. ${ }^{25}$ Atypical ON is due to causes other than MS, presents itself in children under 12 years old or adults over 50 years old, occurring more in men, involvement is bilateral or rapidly sequential, visual loss progresses in two weeks or no recovery within three weeks of the onset of symptoms. It frequently occurs with severe eye pain that wakes them up at night; usually, visual acuity is worse than 20/200 and the ocular fundus shows abnormalities such as significant edema of the optic disc, hemorrhages, exuded, and poor clinical response to steroids; in the orbital magnetic resonance images, there is extensive involvement of the optic nerve, optic chiasm, and optic tracts due to edema and enhancement with the medium of contrast (Table 2).

Atypical ON can be part of the neuromyelitis optica spectrum (NMO), ${ }^{27}$ corticosteroid-dependent chronic relapsing inflammatory optic neuropathy (CRION), and systemic diseases such as sarcoidosis, connective tissue diseases (systemic lupus erythematosus, Sjögren's syndrome, and polyarteritis nodosa), and vasculitis (Wegener's granulomatosis)..$^{25,28,29}$ Others are infectious causes such as Lyme disease, neurosyphilis, tuberculosis, HIV, cytomegalovirus, varicella zoster, toxoplasmosis, and cryptococcosis, among others. A variety of toxins and drugs can also induce optic neuropathy with disc edema, such as lead, phosphodiesterase type 5 inhibitors (PDE5) specific to cyclic guanosine monophosphate and amiodarone..$^{28,30-32}$ Neoplastic processes produce visual disorders and optic neuropathy, whether due to nerve compression, infiltration, increased intracranial pressure (ICP), and paraneoplastic syndromes. Cancers outside of the eyeball and of the nervous system can rarely cause optic neuropathies with disc edema; lung carcinomas are the main cause. Polyneuropathy is often the main symptomatic and disabling feature, but mild visual symptoms occur, and bilateral disc edema is common. ${ }^{33}$

In the specific case of our patient, there was no evidence of clinical activity of melanoma at the level of the central nervous system (CNS) and, on taking the CSF flow cytometry, it reported negative for malignancy. The diagnosis of optic neuritis is clinical, where the supplementary studies are a great aid in the differential diagnosis and in defining the etiological cause. ${ }^{30,31}$ Neural axis resonance imaging, as well as one of the simple orbital and with a medium of contrast, evidenced optic nerve involvement in a percentage greater than $90 \%$; it is useful to evaluate the risk of conversion to multiple sclerosis; however, in our patient, there were red flags to suspect that the etiology of the chart was related to other systemic diseases or, in default of, to drugs. The lumbar puncture, immunological tets, and other extension studies are useful to evaluate possible ischemic, traumatic, toxic, compressive, metabolic, infectious, parainfectious, autoimmune, neoplastic, paraneoplastic, and neuromyelitis optica spectrum causes, especially in cases of atypical ON..$^{28,30,31,32,34}$ For the patient described, additional secondary causes were ruled out after performing test panels of infectious disease, autoimmune, aquaporin 4, simple brain, cervical, and thoracic resonance imaging and with a medium of contrast, normal CSF analysis, and paraneoplastic syndrome. Another test to be considered in assessing these patients, is the optical coherence tomography, a useful test to measure the thickness of the papilla's nerve fiber layer, which at an early stage, can show abnormality in relation to an increase in thickness due to optic nerve edema, and at later stages, there is evidence of a decrease in thickness due to the loss of neurons due to $\mathrm{ON}^{;} ; 3,36$ specifically, in our patient, the test could not be performed due to heavy involvement due to cataracts, which hindered an objective evaluation of the layers of the retina. Visual evoked potentials are useful in cases of diagnostic doubt, where an increase in optic nerve conduction times is relevant, ${ }^{37}$ since this indicates the involvement of the optic pathway, although it does not modify the short-, medium- and long-term prognosis. Under an Optic Neuritis diagnosis, treatment is indicated with high doses of steroids, and in refractory cases, intravenous immunoglobulin is required. In our case, in addition to withdrawing antitumor medication, she received treatment for bilateral optic neuritis with high doses of steroids, as well as plasmapheresis without complications, but without substantial improvement as reported by medical literature. ${ }^{25,28,30,31-34}$

\section{Conclusion}

This patient with metastatic melanoma developed acute vision loss in both eyes, a side effect of longitudinally extensive atypical optic neuritis with bilateral involvement of inflammatory etiology attributed to CTLA-4 blockade related to pembrolizumab treatment. Immune checkpoint inhibitors (ICI) are widely known for the management of melanoma and other types of cancers given their anti-cytotoxic effect associated with CTLA-41,314; by causing an immune system imbalance, adverse effects can be generated that may cause CNS involvement, including the optic nerve. This case emphasizes a rare irAE and refractory, which is why early recognition of adverse events related to the immune system should not be limited to oncologists but should also be extended to general practitioners and physicians from other specialties involved in the care of patients receiving immunomodulatory therapies.

\section{Learning points}

Optic neuritis may have a typical or atypical presentation, the diagnosis is clinical, its differential diagnosis is extensive, its prognosis and treatment depend on the cause.

Cancer patients undergoing treatment with immune checkpoint inhibitors, such as pembrolizumab, may present compromised optic nerves, such as the atypical presentation of optic neuritis.

Anti-PD-1 humanized monoclonal antibodies, such as pembrolizumab and nivolumab, have shown significant antitumor activity in a variety of cancers; however, they are related to the autoimmune destruction of various organs and systems; important side effects to consider

\section{Acknowledgments}

None.

\section{Conflicts of interest}

The author declares that there is no conflict of interest.

\section{References}

1. Sahni S, Valecha G, Sahni A. Role of Anti-PD-1 Antibodies in Advanced Melanoma: The Era of Immunotherapy. Cureus. 2018;10(12):2-10.

2. Siegel R, Miller K, Jemal A. Cancer statistics. CA Cancer J Clin. 2018;(68):7-30

3. Prado G, Svoboda R, Rigel D. What's New in Melanoma. Dermatol Clin. 2019;(37):159-168. 
4. Chapman PB, Hauschild A, Robert $\mathrm{C}$, et al. Improved survival with vemurafenib in melanoma with BRAF V600E mutation. $N$ Engl J Med. 2011;364(26):2507-2516

5. Hodi F, O'Day S, McDermott D, et al. Improved survival with ipilimumab in patients with metastatic melanoma. N Engl J Med. 2010;363(8):711723.

6. Robert C, Thomas L, Bondarenko I, et al. Ipilimumab plus dacarbazine for previously untreated metastatic melanoma. $N$ Engl $\mathrm{J}$ Med. $2011 ;(364): 2517-2526$

7. Falchook GS, Lewis KD, Infante JR, et al. Activity of the oral MEK inhibitor trametinib in patients with advanced melanoma: a phase 1 doseescalation trial. Lancet Oncol. 2012;(13):782-789.

8. Flaherty KT, Infante JR, Daud A, et al. Combined BRAF and MEK inhibition in melanoma with BRAF V600 mutations. $N$ Engl $\mathrm{J} \mathrm{Med}$. 2012;367(18):1694-1703

9. Flaherty KT, Robert C, Hersey P, et al. Improved survival with MEK inhibition in BRAF-mutated melanoma. N Engl J Med. 2012;367(2):107114.

10. Hauschild A, Grob JJ, Demidov LV, et al. Dabrafenib in BRAF-mutated metastatic melanoma: a multicentre, open-label, phase 3 randomised controlled trial. Lancet. 2012;380(9839):358-365.

11. Kim KB, Kefford R, Pavlick AC, et al. Phase II study of the MEK1/MEK2 inhibitor Trametinib in patients with metastatic BRAF-mutant cutaneous melanoma previously treated with or without a BRAF inhibitor. $J$ Clin Oncol. 2013;31(4):482-489.

12. Hamid O, Robert C, Daud A, et al. Safety and tumor responses with lambrolizumab (anti-PD-1) in melanoma. NEngl JMed. 2013;369(2):134 144.

13. Robert C, Ribas A, Wolchok JD, et al. Anti-programmed-death-receptor-1 treatment with pembrolizumab in ipilimumab-refractory advanced melanoma: a randomised dose-comparison cohort of a phase 1 trial. Lancet. 2014;384(9948):1109-1117.

14. Robert $\mathrm{C}$, Long GV, Brady B, et al. Nivolumab in previously untreated melanoma without BRAF mutation. $N$ Engl J Med. 2015;372(4):320-330.

15. Weber JS, D’Angelo SP, Minor D, et al. Nivolumab versus chemotherapy in patients with advanced melanoma who progressed after anti-CTLA-4 treatment (CheckMate 037): a randomised, controlled, open-label, phase 3 trial. Lancet Oncol. 2015;16(4):375-384.

16. Min L, Vaidya A, Becker C. Thyroid autoimmunity and ophthalmopathy related to melanoma biological therapy. Eur J Endocrinol. 2011;164(2):303-307.

17. Beck KE, Blansfield JA, Tran KQ, et al. Enterocolitis in patients with cancer after antibody blockade of cytotoxic T-lymphocyte-associated antigen 4. J Clin Oncol. 2006;24(15):2283-2289.

18. Maker AV, Phan GQ, Attia P, et al. Tumor regression and autoimmunity in patients treated with cytotoxic T lymphocyte-associated antigen 4 blockade and interleukin 2: a phase I/II study. Ann Surg Oncol. 2005;12(12):10051016.

19. Robinson M, Chan C, Yang J, et al. Cytotoxic T lymphocyte-associated antigen 4 blockade in patients with metastatic melanoma: a new cause of uveitis. J Immunother. 2004;27(6):478-479.
20. Kaehler KC, Piel S, Livingstone E, et al. Update on immunologic therapy with anti-CTLA-4 antibodies in melanoma: identification of clinical and biological response patterns, immune-related adverse events, and their management. Semin Oncol. 2010;37(5):485-498.

21. Scarpati G, Fusciello C, Perri F, et al. Ipilimumab in the treatment of metastatic melanoma: management of adverse events. OncoTargets Ther. 2014;(7):203-209.

22. Michot JM, Bigenwald C, Champiat S, et al. Immune-related adverse events with immune checkpoint blockade: a comprehensive review. Eur J Cancer. 2016;(54):139-148.

23. Wilson MA, Guld K, Galetta S, et al. Acute visual loss after ipilimumab treatment for metastatic melanoma. J Immunother Cancer. 2016;4(1):66.

24. Weber J, Kudchadkar R, Yu B, et al. Safety, efficacy, and biomarkers of nivolumab with vaccine in ipilimumab-refractory or -naive melanoma. $J$ Clin Oncol. 2013;31(34):4311-4318.

25. Abel A, McClelland C, Lee M. Critical review: Typical and atypical optic neuritis. Survey Of Ophthalmology. 2019;64(6):770-779.

26. Wakakura $\mathrm{M}$, Ishikawa $\mathrm{S}$, Oono $\mathrm{S}$, et al. Incidence of acute idiopathic optic neuritis and its therapy in Japan. Optic Neuritis Treatment Trial Multicenter Cooperative Research Group (ONMRG). Nippon Ganka Gakkai Zasshi. 1995;99(1):93-97.

27. Orman G, Wang K, Pekcevik Y, et al. Enhancing Brain Lesions during Acute Optic Neuritis and/or Longitudinally Extensive Transverse Myelitis May Portend a Higher Relapse Rate in Neuromyelitis Optica Spectrum Disorders. AJNR Am J Neuroradiol. 2017;38(5):949 -953.

28. Toosy A, Mason D, Miller D. Optic neuritis. Lancet Neurol. 2014;13(1):83-

29. De la Cruz J, Kupersmith M. Clinical profile of simultaneous bilateral optic neuritis in adults. Br J Ophthalmol. 2006;90(5):551-554.

30. Balcer L. Clinical practice. Optic neuritis. $N$ Engl $J$ Med 2006;354(12):1273-1280.

31. Hoorbakht H, Bagherkashi F. Optic Neuritis, its Differential Diagnosis and Management. Open Ophthalmol J. 2012;(6):65-72.

32. Drori T, Cahpman J. Diagnosis and classification por neuromylitis óptica (Devic's Syndrome). Autoimmunity reviews. 2014;13(4-5)531-533.

33. Cohen A, Pless M. Optic neuropathy With Disc Edema. American academy neurology. 2009;15(4):26-46.

34. Frohman E, Frohman T, Zee D, et al. The neuro-ophthalmology of multiple Sclerosis. Lancet Neurol. 2005;4(2):111-121.

35. Kallenbach K, Frederiksen J. Optical coherence tomography in optic neuritis and multiple sclerosis: a review. Eur J Neurol. 2007;14 (8):841849

36. Vidal A, Sastre J, Montalban X. Optical coherence tomography in multiple sclerosis. Rev Neurol. 2012;54(9):556-563.

37. Kolappan M, Henderson A, Jenkins T, et al. Assessing structure and function of the afferent visual pathway in multiple sclerosis and associated optic neuritis. J Neurol. 2009;256(3):305-319.

38. Greenwald R, Freeman G, Sharpe A. The B7 family revisited. Annu Rev Immunol. 2005,(23):515-548. 